\title{
Isolated fallopian tube torsion with paraovarian cysts: a case report and literature review
}

\author{
Liang Qian ${ }^{1}$, Xue Wang ${ }^{2}$, Dingheng $\mathrm{Li}^{{ }^{*}}{ }^{\mathbb{D}}$, Songyi $\mathrm{Li}^{1}$ and Jiashan Ding ${ }^{3}$
}

\begin{abstract}
Background: Isolated fallopian tube torsion (IFTT) is a rare cause of gynecological acute abdomen, is easily misdiagnosed and often has a delay in diagnosis. IFTT with paraovarian cysts is most frequently reported in studies. Here, we reported a patient diagnosed with IFTT associated with a paraovarian cyst, and we conducted a literature review for IFTT, aiming to identify valuable information that will be helpful for diagnosis and treatment for fallopian tube torsions.
\end{abstract}

Case presentation: A 13-year-old girl presented with a 10-day history of right lower abdominal pain that worsened 2 days before presentation. On presentation, ultrasound showed a $5.8 * 5.5 \mathrm{~cm}$ hypoechoic cyst adjacent to the right ovary, and between the cyst and ovary, a tortuous thickened tube was visualized. Laparoscopy revealed a triple torsion of the right fallopian tube with a 6-cm paraovarian cyst, and tubal conservation surgery was performed. The postoperative course was uneventful. Histopathological diagnosis revealed serous papillary cystadenoma.

Conclusion: Paraovarian cystic dilatation often occurs in adolescence and can induce fallopian torsion when the size of the cyst reaches $5-\mathrm{cm}$. In our review, the median age of patients diagnosed with IFTT with paraovarian cysts was 15 years old, and the main clinical manifestation was emergency abdominal pain. The associated symptoms were variable, and vomiting was the most commonly associated symptom. Salpingectomy was the most common procedure performed; however, timely surgical intervention can effectively avoid salpingectomy.

Keywords: Case report, Isolated fallopian tube torsion, Paraovarian cyst, Salpingectomy, Ultrasound

\section{Background}

IFTT is the torsion of the fallopian tube on its own axis. It is a rare cause of gynecological acute abdomen and was first reported by Bland-Sutton [1]. The estimated prevalence is 1 in 1.5 million women [2]. IFTT is easy to misdiagnosis, and treatment can be delayed due to its rarity of occurrence and nonspecific clinical manifestation. The etiologies for IFTT include intrinsic and extrinsic factors such as anatomic abnormalities, sudden changes in

\footnotetext{
*Correspondence: lidh2016@126.com

1 Department of Gynecology, Hangzhou Women's Hospital,

Hangzhou 310008, Zhejiang, China

Full list of author information is available at the end of the article
}

body position, hyperlaxity of the tube and ligament due to gestation or acyeterion drugs, adhesions due to pelvic inflammation and so on [3]. Anatomic abnormalities, including hydrosalpinx, hematosalpinx, paraovarian cyst, tubal tumor, long tubal, and paraovarian cyst, were most frequently reported in previous studies. However, to the best of our knowledge, no systematic review of IFTT with paraovarian cysts currently exists. Herein, in this study, we reported a case of a 13-year-old girl diagnosed with IFTT with a paraovarian cyst and conducted a literature review on this patient with IFTT. The purpose of this study was to determine valuable information that could be helpful for the diagnosis and treatment of fallopian tube torsions. 


\section{Case presentation}

A 13-year-old sexually inactive girl was admitted to our department due to a 10-day history of right lower abdominal pain, which worsened the last 2 days before presentation. It was suspected that she had an ovarian torsion and was treated by a local hospital with medication that did not relieve the symptoms. The physical examination suggested lower abdominal tenderness without defense. Pelvic examination revealed normal external genitalia. A cystic mass approximately $6-\mathrm{cm}$ in diameter was palpable behind the uterus. Laboratory evaluations showed normal blood tests. Transabdominal and transrectal ultrasounds showed normal ovaries and a $5.8 * 5.5 \mathrm{~cm}$ hypoechoic cyst adjacent to the right ovary, and between the cyst and ovary, a tortuous thickened tube was visualized (Fig. 1A, B). Furthermore, a two-mm papillary projection was also seen on the inner wall of the cyst (Fig. 1C).

A preliminary diagnosis of IFTT was made, and laparoscopy was performed immediately, which showed a triple torsion of the right fallopian tube associated with a six-cm paraovarian cyst. The fallopian tube appeared dilated and edematous. After the detorsion of the fallopian tube and denucleating the cyst, the right fallopian tube return to a pink color after reperfusion (Fig. 2). The postoperative course was uneventful, and she was discharged 5 days after the operation. The histopathological diagnosis was serous papillary cystadenoma (Fig. 3).

We searched the PubMed database by using the terms "paratubal cyst", "paraovarian cyst", "tubal torsion", "isolated fallopian tube torison", and "isolated tubal torsion". After screening all of the publications, cases of IFTT associated with paraovarian cysts written in English were included in the analysis. References without a fulltext article were excluded. The details of the patients' diagnosis and treatment processes, such as age, presentation, degree of pain, blood tests, preoperative diagnosis, surgical approach, and torsion time, were collected and analyzed.

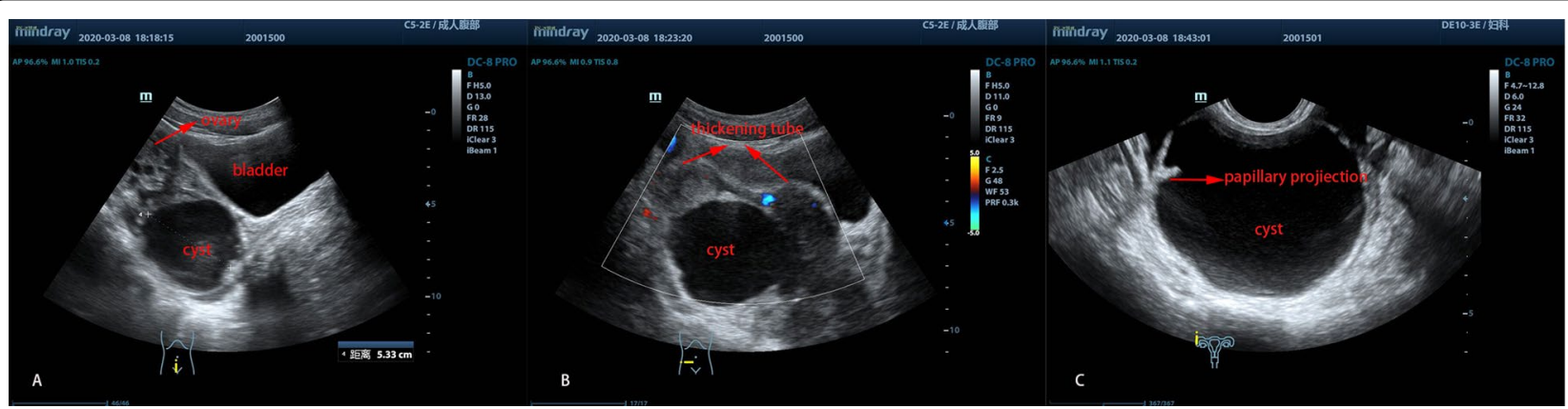

Fig. 1 Ultrasonographic identification of a cystic mass in the right lower abdomen $\mathbf{A}$ a normal ovary with a cystic mass underneath it. B Thickening tube beside the cyst. C The papillary projection in the cyst wall

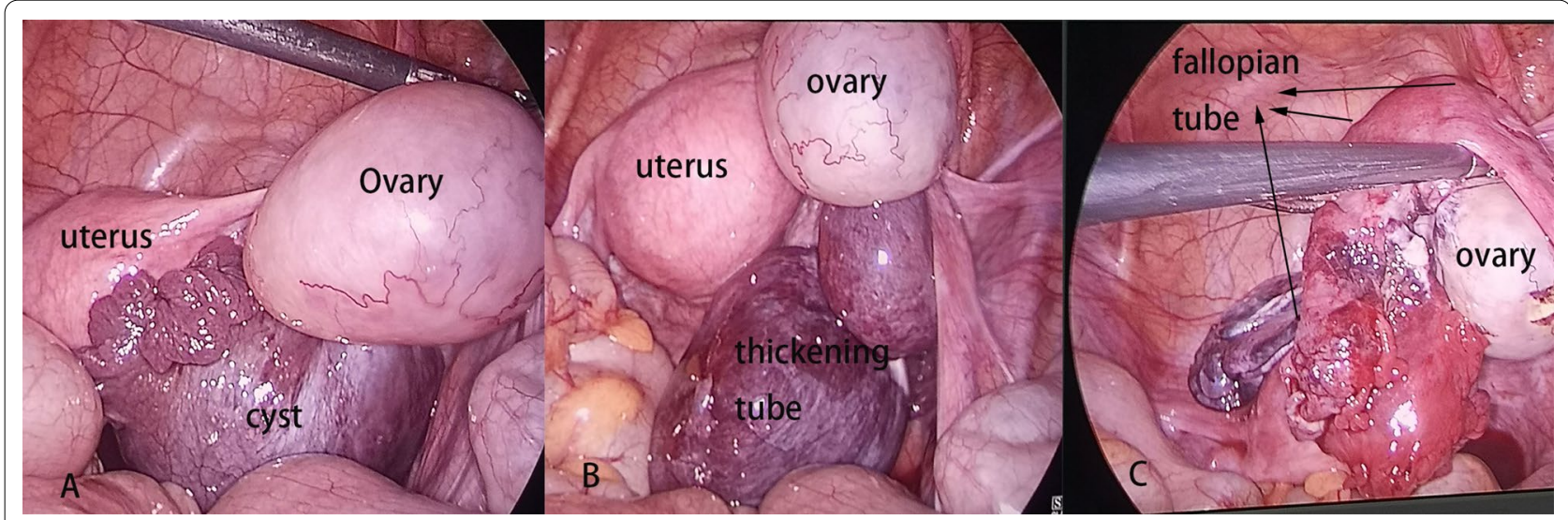

Fig. 2 A The normal ovary and uterus, with a cystic mass in the middle. B The thickening of the torsed tube. C The fallopian returned to a pink color after the detorsion of the tube and denucleating the cyst 


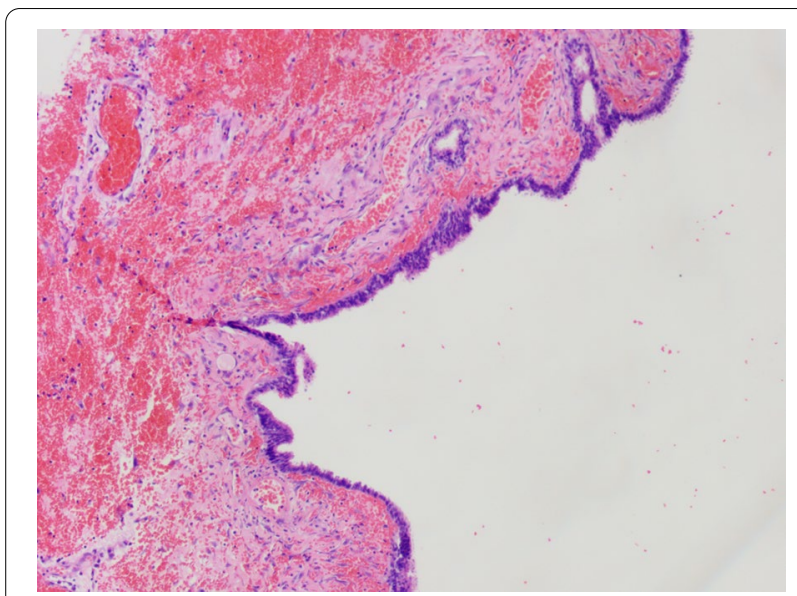

Fig. 3 Photomicrograph of the cyst shows serous papillary cystadenoma, accompanied by hemorrhagic infarct on the wall. Magnification $\times 100$

\section{Results}

After the literature search on PubMed, this study included 19 publications regarding IFTT with paraovarian cysts. Among these studies, 17 were case reports (Table 1), and another two were case series without details of the patients $[4,5]$. We analyzed 20 patients included for our study. The median age of patients was 15 years old. Four patients had a torsion of the left fallopian tube, while 14 patients had a torsion of the right fallopian tube. Another two patients had bilateral torsions. The mean number of torsion rotations was 2.38 rotations, ranging from one to four rotations. All patients reported abdominal pain, 17 cases had the mode of pain reported, and all patients presented with emergency abdominal pain. The median pain duration time was 3 days (the longest 5 months, the least $4 \mathrm{~h}$ ). Nine patients presented with accompanying symptoms of vomiting. Micturition abnormalities $(\mathrm{n}=1)$, dyspareunia $(\mathrm{n}=1)$, tachycardia $(n=1)$, and diarrhea $(n=1)$ were also present. Except for two previous cases from 1967 and 1972, ultrasonography was performed as the first diagnostic examination in all other cases.

Blood tests were reported in 16 patients, six patients showed leukocytosis, and one patient showed elevated CRP and CA199. Nineteen patients had the surgical approaches recorded, and these included 13 laparoscopic surgeries and six laparotomies. The most common procedure performed (13 cases) was salpingectomy, and one of the procedures was a radical operation performed due to malignant paraovarian cancer found by rapid pathological examination. Conservative surgical management was performed in six out of 19 cases, in which the salpinges appeared viable after detorsion. Histopathologic diagnosis was reported in 17 cases; only six cases had definitive diagnoses, including three cases of mesothelial origin, one case of mesonephric origin and two cases of paramesonephric origin.

\section{Discussion and conclusions}

Paraovarian or paratubal cysts are present in approximately $10 \%$ of all adnexal masses [6]. They arise from epoophores, which are located in the broad ligament and consist of a longitudinal ductulus and 10-20 transverse ductuluses, and these ductuli are secretory (Fig. 4). Paraovarian cysts should be distinguished from Morgagni's hydatid cysts, which are usually smaller in size and located in the fimbriated end of the fallopian tube. Paraovarian cysts are generally unilocular, contain clear fluid and are of paramesonephric, mesothelial, or mesonephric origin [7]. Histopathology may show secretory and ciliated cells (paramesonephric origin), low cuboidal epithelium and occasional clear cells (mesonephric origin), lined by flattened epithelium with occasional tubal differentiation and surrounding fibrous tissue (mesothelial origin). Because distention of the cavity often distorts the epithelium, absolute differentiation is difficult [8]. According to the results of this study, only $6 / 20$ cases determined the origins of the cysts.

In general, parovarian cysts could be found at any age, and most of them were benign tumors. Paraovarian cysts are usually detected by ultrasonography or during surgery. Nevertheless, due to hormonal activity, especially in adolescence or during pregnancy, cystic dilatation often occurs [9]. In this study, we found that the average diameter of cysts when torsion occurred was approximately $5-\mathrm{cm}$ (the minimum was $2-\mathrm{cm}$, and the maximum was 9-cm); the median age was 15 years; $13 / 20$ of the patients were adolescents; $6 / 20$ patients were adults; and $1 / 20$ was postmenopausal. In addition, $5 / 20$ cases had torsions that occurred during pregnancy (four cases) or puerperium (one case). Cystic distention could increase the mobility of adnexal tissues, which may induce a reversal, particularly when the cysts reach $5-\mathrm{cm}[10]$. The cysts can torse on their own or can predispose to isolated tubal torsion. The distinction between the two situations before surgery is challenging, and the main clinical manifestation is abdominal pain.

Patients presented with abdominal pain, which could be described as gradual or acute, persistent or intermittent, and mild or severe. We found that 17 cases had the mode of pain recorded, and all of them presented with emergency abdominal pain, such as acute pain, severe pain, and aggravated pain. Some of the cases had a history of previous mild pain that subsequently worsened. Associated symptoms were variable and the most frequent symptom was vomiting $(9 / 20)$, which was considered a stress response to the intense abdominal pain. On 


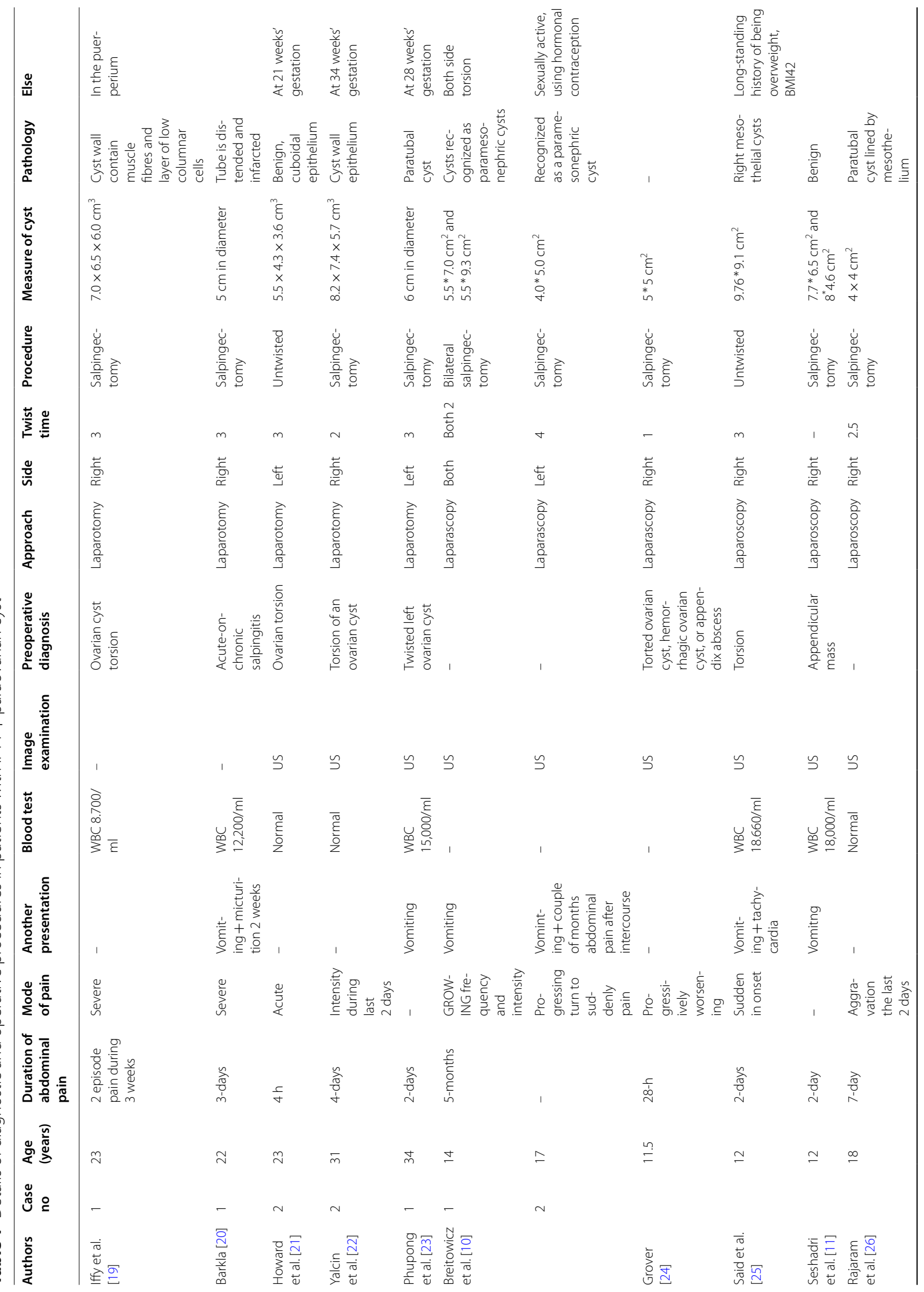




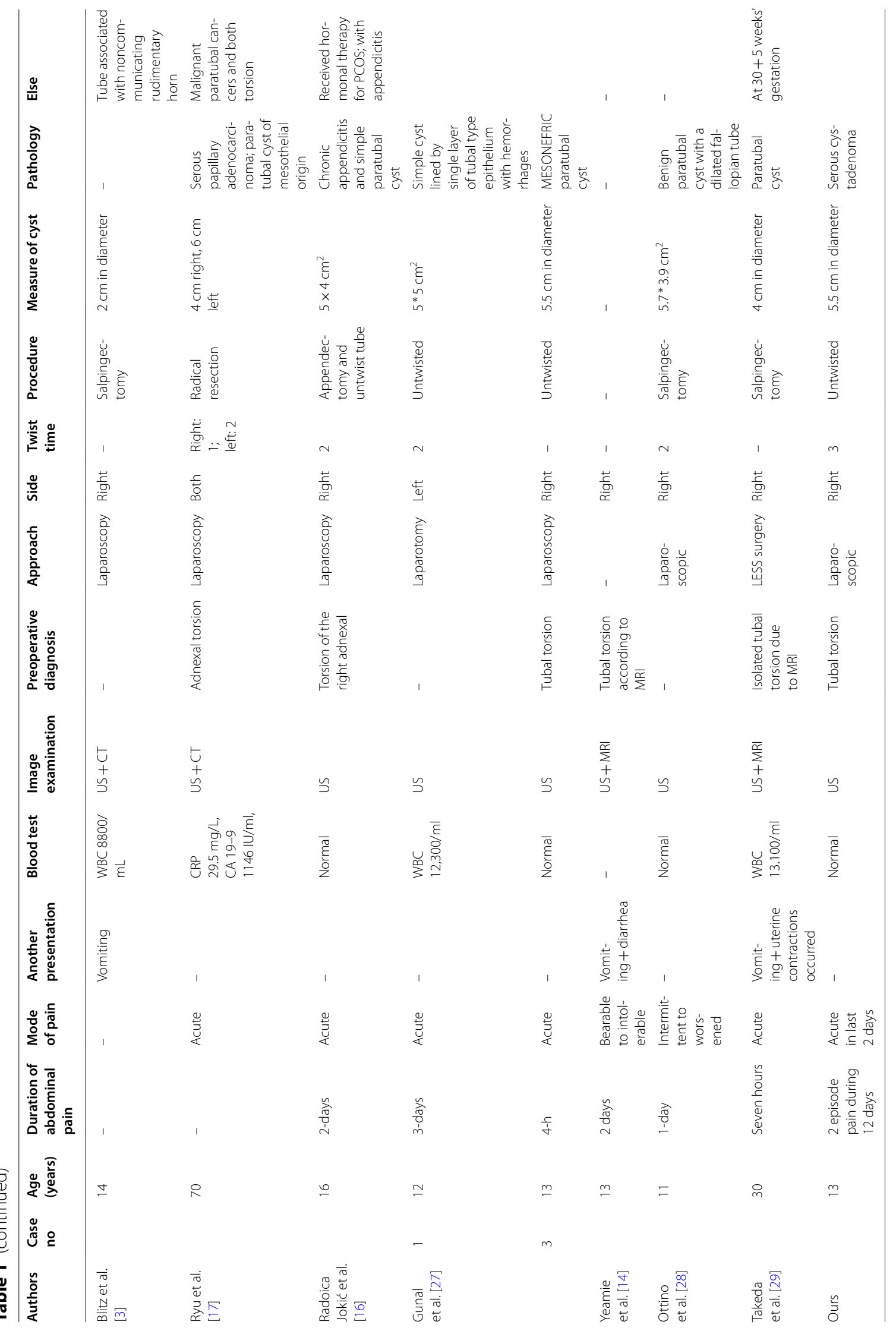




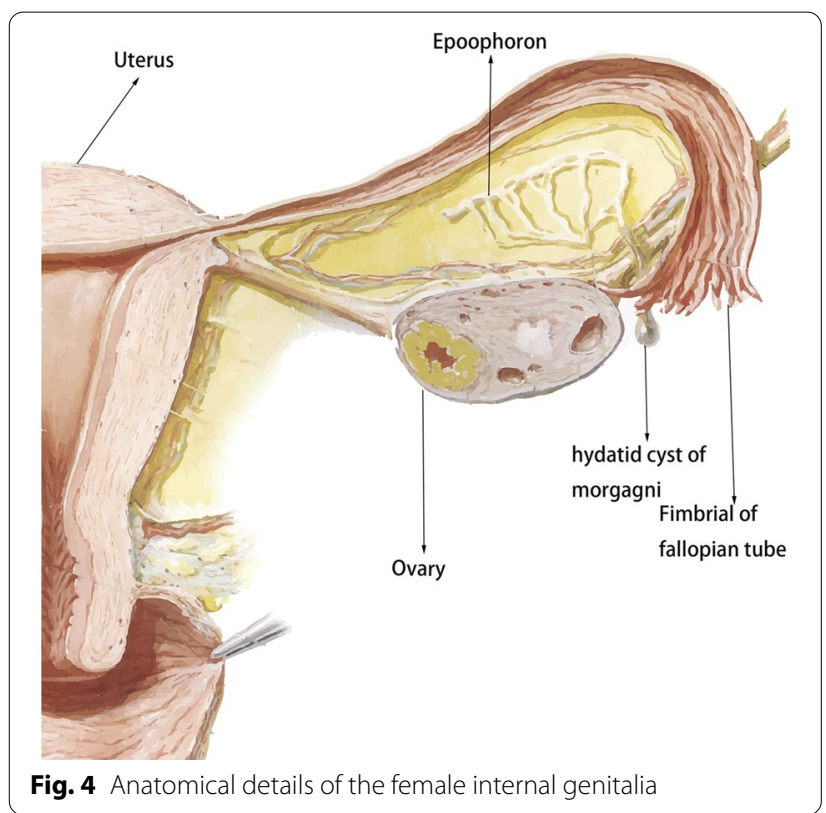

physical examination, mild tenderness was present in all patients, and peritoneal signs developed in advanced cases (if the condition progressed) [11]. The median duration time of pain was 3 days. Mazouni et al. [12] reported that the risk of adnexal necrosis was increased significantly when the delay time for surgery was over $10 \mathrm{~h}$. A previous study also found that patients with pain for more than $24 \mathrm{~h}$ were more likely to undergo salpingectomy, suggesting that longer periods of torsion can lead to more tissue necrosis [5]. Moreover, we observed that nine patients presented with vomiting, $8 / 9$ patients had their procedure reported, and 7/8 patients underwent salpingectomy. This may demonstrate that the amount and severity of necrosis from the torsion is related not only to the duration of pain but also to the degree of pain, which probably represents the varying degrees of torsion. Until now, most importantly, timely diagnosis and surgical intervention have remained the most effective ways to avoid salpingectomy.

The majority of fallopian tube torsions occurred on the left $(14 / 20)$. The most likely explanation was that the sigmoid colon on the left provides cushioning as an accessory to prevent torsion by limiting the torsional activity, and patients with right abdominal pain more often underwent surgery because of the suspicion of appendicitis [13]. Fifteen of 20 cases had the number of torsion rotations recorded, and the average number of torsion rotations was 2.38 rotations. There was no significant difference in torsion time between the salpingectomy group and preservation group $(P=0.651)$, demonstrating that necrosis was not associated with torsion times.

Ultrasonography was performed as the first diagnostic examination in all cases. The most specific characteristic of imaging of IFTT with paraovarian cysts was the presence of normal ovaries and a cystic mass, with or without a dilatated fallopian tube mass, which was due to tubal edema due to the torsion. The "break sign" was also another specific sign of IFTT, which showed narrowing at the extremity of the tube due to the torsion [14]. Color Doppler ultrasonography can detect tubal blood flow, which can also be detected in cases of incomplete obstruction, so the presence of flow cannot completely rule out torsion. However, a high impedance waveform with reversal of diastolic flow could be helpful, which implies torsion of the tube [15]. Compared with ultrasound, MRI could more clearly show findings such as dilated tubes, beak signs, and twisted pedicles. In this study, two patients were diagnosed by MRI as having IFTT with paratubal cysts before surgery. Radoica Jokić et al. [16] reported that MRI and ultrasound could provide credible information without the risks of radiation, which is especially helpful for pregnant patients. If a papillary projection on the cyst wall is present in sonographic or MRI images, malignant tumors and cystadenomas should be considered despite the low incidence of paraovarian cystic cancers [17].

In this study, most patients (13/19) underwent salpingectomy, and only six patients underwent more conservative surgical management, including detorsion of the tube and removal of paraovarian cysts. Surgical decisions depended on the observations of surgeons during the operation and whether the tubes developed normal circulation without necrosis after detorsion. Bertozzi et al. [18] reported that conservative management for IFTT could also be considered in cases of necrotic tubes because morbidity would not increase, but it could create the possibility of leaving a nonfunctional tube. Considering that the majority of patients were adolescents, tubal conservation should be favored as much as possible, because of the concerns for fertility in the future.

\section{Abbreviations}

IFTT: Isolated fallopian tube torsion; ICU: Intensive care unit.

\section{Acknowledgements}

Not applicable.

\section{Authors' contributions}

L-Q: project development, data collection, and manuscript writing and review. DH-L: project development, manuscript writing and review. SY-L: project development, data collection, manuscript review. X-W: data analysis, manuscript review, improvements to the English language. JS-D: project 
development, data collection, manuscript review. All authors read and approved the final manuscript.

\section{Funding}

Medical Health Science and Technology Project of Zhejiang provincial commission to Dr. Dingheng Li (2018248116): Design of study and analysis, writing the manuscript. Medical Health Science and Technology Project of Zhejiang provincial commission to Dr. Songyi Li (2017KY553): Interpretation of data and writing the manuscript.

\section{Availability of data and materials}

All data generated or analyzed during this study are included in this published article.

\section{Declarations}

\section{Ethics approval and consent to participate}

Not applicable.

\section{Consent for publication}

Written consent was obtained from the patient's parents for publication of clinical information and photographs.

\section{Competing interests}

The authors declare that they have no competing interests.

\section{Author details}

'Department of Gynecology, Hangzhou Women's Hospital, Hangzhou 310008, Zhejiang, China. ${ }^{2}$ Nanjing Medical University, Nanjing 211100, Jiangsu, China. ${ }^{3}$ Department of Obstetrics and Gynecology, The First Affiliated Hospital of Nanchang University, Nanchang 330006, China.

\section{Received: 6 December 2020 Accepted: 17 September 2021}

\section{Published online: 28 September 2021}

\section{References}

1. Bland-Sutton J. Salpingitis and some of its effects. Lancet. 1890;2:1206-9.

2. Shukla R. Isolated torsion of the hydrosalpinx: a rare presentation. $\mathrm{Br} \mathrm{J}$ Radiol. 2004;77(921):784-6.

3. Blitz MJ, Appelbaum H. Torsion of fallopian tube remnant associated with noncommunicating rudimentary horn in adolescent girl with unicornuate uterus. J Pediatr Adolesc Gynecol. 2014;27(5):e97-9.

4. Alpern MB, Sandler MA, Madrazo BL. Sonographic features of parovarian cysts and their complications. AJR Am J Roentgenol. 1984;143(1):157-60.

5. Webster KW, Scott SM, Huguelet PS. Clinical predictors of isolated tubal torsion: a case series. J Pediatr Adolesc Gynecol. 2017;30(5):578-81.

6. Kiseli M, Caglar GS, Cengiz SD, et al. Clinical diagnosis and complications of paratubal cysts: review of the literature and report of uncommon presentations. Arch Gynecol Obstet. 2012;285(6):1563-9.

7. Salamon C, Tornos C, Chi DS. Borderline endometrioid tumor arising in a paratubal cyst: a case report. Gynecol Oncol. 2005;97(1):263-5.

8. Genadry R, Parmley T, Woodruff JD. The origin and clinical behavior of the parovarian tumor. Am J Obstet Gynecol. 1977;129(8):873-80.

9. Dietrich JE, Adeyemi O, Hakim J, et al. Paratubal cyst size correlates with obesity and dysregulation of the Wnt signaling pathway. J Pediatr Adolesc Gynecol. 2017;30(5):571-7.

10. Breitowicz B, Wiebe BM, Rudnicki M. Torsion of bilateral paramesonephric cysts in young girls. Acta Obstet Gynecol Scand. 2005;84(2):199-200.
11. Seshadri S, Morris A, Uchil D, et al. Bilateral paratubal cysts with coexistent fallopian tube torsion in an adolescent. J Obstet Gynaecol. 2009:29(6):564-5.

12. Mazouni C, Bretelle F, Menard JP, et al. Diagnosis of adnexal torsion and predictive factors of adnexal necrosis. Gynecol Obstet Fertil. 2005;33(3):102-6.

13. Harmon JC, Binkovitz LA, Binkovitz LE. Isolated fallopian tube torsion: sonographic and CT features. Pediatr Radiol. 2008;38(2):175-9.

14. Kousari YM, Pollock AN. Isolated fallopian tube torsion with paraovarian cyst. Pediatr Emerg Care. 2016;32(11):817-9.

15. Perlman S, Hertweck P, Fallat ME. Paratubal and tubal abnormalities. Semin Pediatr Surg. 2005;14(2):124-34.

16. Jokic R, Lovrenski J, Lovrenski A, et al. Isolated fallopian tube torsion-a challenge for the timely diagnosis and treatment. Srp Arh Celok Lek. 2015;143(7-8):471-5.

17. Ryu KJ, Kim IS, Bae HS, et al. Paratubal cancer found at the time of laparoscopic surgery for adnexal torsion: a case report and literature review. Eur J Gynaecol Oncol. 2014;35(6):741-4.

18. Bertozzi M, Magrini E, Riccioni S, et al. Isolated fallopian tube torsion with hydrosalpinx: review of a debated management in a pediatric population. J Pediatr Surg. 2017;52(10):1553-60.

19. Iffy L, Adolph J. Twisted parovarian cyst in the puerperium: report of a case. Can J Surg. 1967;10(3):345-7.

20. Barkla PC, Hammill PH. Torsion of fallopian tube. Lancet. 1972;1(7753):745

21. Howard FM, Vill M. Laparoscopic adnexal surgery during pregnancy. J Am Assoc Gynecol Laparosc. 1994;2(1):91-3.

22. Yalcin OT, Hassa H, Zeytinoglu S, et al. Isolated torsion of fallopian tube during pregnancy; report of two cases. Eur J Obstet Gynecol Reprod Biol. 1997;74(2):179-82.

23. Phupong $V$, Intharasakda P. Twisted fallopian tube in pregnancy: a case report. BMC Pregnancy Childbirth. 2001;1(1):5

24. Grover S. Torsion causing interruption of the ampullary portion of the fallopian tube. Fertil Steril. 2007;88(4):913-68.

25. Said MR, Bamigboye V. Twisted paraovarian cyst in a young girl. J Obstet Gynaecol. 2008;28(5):549-50.

26. Rajaram S, Bhaskaran S, Mehta S. Isolated fallopian tube torsion in adolescents. Case Rep Obstet Gynecol. 2013;2013:341507

27. Gunal YD, Bahadir GB, Boybeyi $O$, et al. A rare cause of acute abdominal pain in children: isolated tubal torsion; a case series. Turk J Emerg Med. 2017;17(2):73-6.

28. Ottino J, Ricca R. Paratubal cyst torsion with compromise of the fallopian tube in an adolescent girl. Am Surg. 2016;82(9):777-8.

29. Takeda A, Kitami K, Shibata M. Magnetic resonance imaging and gasless laparoendoscopic single-site surgery for the diagnosis and management of isolated tubal torsion with a paratubal cyst at 31 weeks of gestation: a case report and literature review. J Obstet Gynaecol Res. 2020;46(8):1450-5.

\section{Publisher's Note}

Springer Nature remains neutral with regard to jurisdictional claims in published maps and institutional affiliations.

Ready to submit your research? Choose BMC and benefit from

- fast, convenient online submission

- thorough peer review by experienced researchers in your field

- rapid publication on acceptance

- support for research data, including large and complex data types

- gold Open Access which fosters wider collaboration and increased citations

- maximum visibility for your research: over $100 \mathrm{M}$ website views per year

At $\mathrm{BMC}$, research is always in progress.

Learn more biomedcentral.com/submissions 